\title{
Standard KDFOC4 Fallout Calculations for Buried Nuclear Detonations
}

\author{
F. Serduke
}

September 14, 2001

U.S. Department of Energy

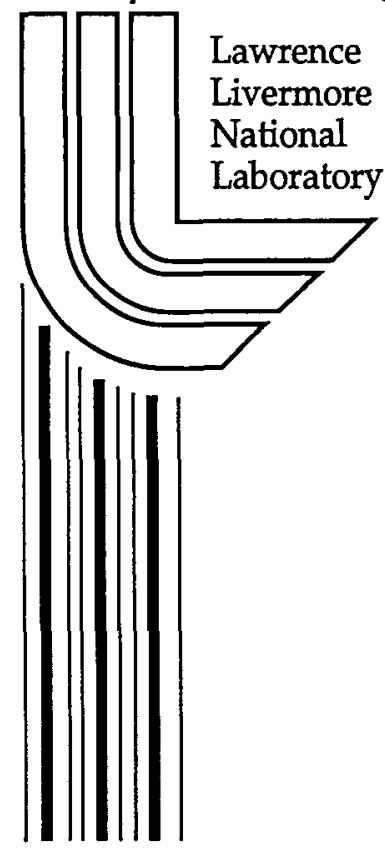




\section{DISCLAIMER}

This document was prepared as an account of work sponsored by an agency of the United States Government. Neither the United States Government nor the University of California nor any of their employees, makes any warranty, express or implied, or assumes any legal liability or responsibility for the accuracy, completeness, or usefulness of any information, apparatus, product, or process disclosed, or represents that its use would not infringe privately owned rights. Reference herein to any specific commercial product, process, or service by trade name, trademark, manufacturer, or otherwise, does not necessarily constitute or imply its endorsement, recommendation, or favoring by the United States Government or the University of California. The views and opinions of authors expressed herein do not necessarily state or reflect those of the United States Government or the University of California, and shall not be used for advertising or product endorsement purposes.

This work was performed under the auspices of the U. S. Department of Energy by the University of California, Lawrence Livermore National Laboratory under Contract No. W-7405-Eng-48.

This report has been reproduced directly from the best available copy.

Available electronically at http://www.doc.gov/bridge

Available for a processing fee to U.S. Department of Energy

And its contractors in paper from

U.S. Department of Energy

Office of Scientific and Technical Information

P.O. Box 62

Oak Ridge, TN 37831-0062

Telephone: (865) 576-8401

Facsimile: (865) 576-5728

E-mail: reports@adonis.osti.gov

Available for the sale to the public from

U.S. Department of Commerce

National Technical Information Service

5285 Port Royal Road

Springfield, VA 22161

Telephone: (800) 553-6847

Facsimile: (703) 605-6900

E-mail: orders@ntis.fedworld.gov

Online ordering: http://www.ntis.gov/ordering.htm

OR

Lawrence Livermore National Laboratory

Technical Information Department's Digital Library

http://www.llnl.gov/tid/Library.html 


\section{Introduction:}

The collateral damage caused by fallout from shallow-buried nuclear devices is of considerable interest. In this paper, we present results for "standard" calculations using the KDFOC4 fallout computer code. Results are presented for a parametric range of yields from $0.1 \mathrm{kt}$ to $1 \mathrm{Mt}$ in equally-spaced logarithmic increments and for emplacement depths of 5 meters in hard, dry rock and 20 meters in moist soil. We will see that for low yields, this emplacement depth has a marked influence on the shape of the fallout patterns but for the highest yields, the fallout patterns are insensitive to the emplacement medium and depth. We look at two categories of doses: 1) Those for which health effects begin to be serious and range upward to lethal, and 2) Doses that are politically very sensitive but for which any deleterious health effects are difficult to prove.

\section{KDFOC4 Fallout Calculations:}

The KDFOC4 code is the code recommended by the Defense Threat Reduction Agency (formerly the Defense Nuclear Agency) as the best code for predicting buried-burst nuclear fallout. This code was developed at LLNL by Ted Harvey in collaboration with the author (refs. 1 and 2). The parametric range of fission yields selected for this set of calculations was $0.1,0.3,1,3,10,30,100,300$, and 1000 kilotons. The doses that were deemed of interest are discussed below. To illustrate the effect of emplacement medium on the results, emplacement depths of 5 meters in rock and 20 meters in soil were chosen. The winds used for all of the calculations were the same default wind field contained in KDFOC4. It is a moderate speed wind with modest shear, both in direction and speed.

Note: none of the detonations is even approximately fully contained.

\section{Fallout Doses:}

Several radiation doses were selected. High 24 hour doses and low 7 day doses were chosen as it was felt that these choices might reflect the time an individual might be compelled to remain in a fallout field.

450 rem: This is regarded as a lethal dose to $50 \%$ of the population. It is called the LD50 dose.

4500 rem: This dose would be received in radioactively hot regions and might be construed as a region that is denied to locals for a period of time after the detonation because of the high doses. Individuals in the basements of homes receive a radiation protection factor of approximately 10 . Thus, the 4500 rem contour would encompass the LD50 dose for individuals who are sheltered as well as they might be able to achieve on their own and who do not leave the area in directions of lower exposures.

100 rem: At 100 rem, radiation sickness symptoms begins to manifest themselves. It is roughly in this neighborhood that dose is lethal to some weaker individuals of the population. Similar statements have been made about 50 rem and 150 rem doses.

5 rem: This is the dose that the U.S. government permits its radiation workers to receive over a period of 1 year. Arguably, this dose would have no effect on healthy individuals. 
0.2 to 0.4 rem: This is the neighborhood of the annual natural background dose that everyone receives by dint of living on this planet. Depending on the device yield, a 7-day fallout dose is approximately $2 / 3$ of the dose that would be received if an individual never left the area.

\section{Results of Calculations:}

Figures 1 and 2 summarize the areas and down-wind distances of the fallout contours for doses ranging from 4500 rem in 24 hour to $0.2 \mathrm{rem}$ in one week where the device is in 20 meters of soil. Results for the areas and down-wind distances of the fallout contours of device emplacement under 5 meters of hard, dry rock are presented in figures 3 and 4. For comparison purposes, the area plots in figures 1 and 3 are combined in figure 5 and the down-wind distance plots from figures 2 and 4 are presented in figure 6 . The colors in figures 5 and 6 mean the same as they do in the first four figures; the $5 \mathrm{~m}$ Rock results are plotted with thick lines and the $20 \mathrm{~m}$ Soil results are presented as thin lines. Results on these log-log plots look quite similar but differences of roughly a factor-of-two are seen in the comparisons. One point to be observed is that with a deeper burial, the local fallout becomes more local or comes down closer to ground zero. This shows itself in the areas in figure 5 where the thin lines (soil at 20 meters) are generally above the thick lines of the same color for the high doses but for the lowest, sub-1-rem doses which extend to far larger distances, the fallow contours for detonations in $5 \mathrm{~m}$ of rock extend beyond those of the deeper soil.

The impact of fallout on populations depends on the doses received both by individuals and by the population as a whole. A zero order assessment would involve the use of the areas of the fallout contours and their overlap with populated areas. The fallout areas for the selected doses are presented in square kilometers. Urban population densities are of order 10,000 persons per square kilometer. Some small areas of New York have population densities over 50,000 per square kilometer. The average population density of the greater Calcutta, India region is about 25,000 per square kilometer. Suburban population densities in the United States are in the neighborhood of 1000 per square kilometer give or take a factor of few. The U.S. Census bureau loosely defines the "urban fringe" as having a population density of about 400 per square kilometer; there are political implications in their choice and there are lots of qualifiers appended to this number.

Fallout patterns of the high, health-affecting 24-hour doses for the extremes of our set of yields are presented in figures 7 and 8.

Figure 7 shows the fallout patterns for a $0.1 \mathrm{kt}$ detonation emplaced in $5 \mathrm{~m}$ of rock and $20 \mathrm{~m}$ of soil. The detonation in soil is near the optimum cratering depth and this is the situation in our calculations that most nearly approaches containment. If the detonation were somewhat deeper than $\mathbf{3 0}$ meters below the surface, this detonation might well form only a retarc (crater spelled backwards) which is a mound of soil pushed up by the detonation. The greater width of the fallout pattern for the $0.1 \mathrm{kt}$ at a depth of $20 \mathrm{~m}$ is due to the so-called base-surge cloud that forms when a dense cloud of ejecta is thrown upward, settles to the surface and roils outward carrying radioactive debris horizontally in 
all directions. Although a base surge does form at the shallower 5 meter depth of burst, its effect is smaller.

Figure 8 presents the results for a one-megaton detonation in our 5-meter rock and 20meter soil scenarios. The lethal-dose contours extend over 100 kilometers and the region that would lead to radiation-sickness symptoms extends over 200 kilometers. At one megaton, the emplacement depth and medium have only minimal effect on the fallout pattern.

\section{Summary and Conclusions:}

We have presented KDFOC4 fallout results for shallow-buried bursts for a wide range of nuclear yields. The KDFOC4 code uses a single wind and assumes a flat terrain. These limits on its capabilities could be significantly improved by incorporating the KDFOC4 source term together with its dispersion and settling velocity procedures into a more sophisticated atmospheric transport code such as those used by the NARAC facility at LLNL. This would also provide access to terrain information and population databases that would be extremely useful in carrying out assessments of collateral damage. The current results are probably reasonable for flat terrain for this range of yields. The predictions become less reliable when low yields are detonated in mountainous regions. High-yield fallout patterns would be less influenced by terrain because the nuclear cloud would carry radiation to altitudes well above the terrain features.

The present results summarize the extent of the regions of serious health effects for the fallout scenarios considered; areas range from a few to several thousand square kilometers and the down-wind extent of these regions goes from a few to several hundred kilometers from ground zero. The very modest dose contours have areas nearly two orders of magnitude larger and extents about one order of magnitude larger; these are potentially extremely sensitive politically.

\section{References:}

1) T. Harvey, F. Serduke, and L. Peters "KDFOC3: A Nuclear Fallout Assessment Capability" LLNL Report UCRL-52338, September 13,1994

2) Ted Harvey, Bob Fries, Doug Lohafer, Frank Serduke, Alan Moore "KDFOC4.1 User's Manual Including Test Cases" LLNL Report UASG 98,-11 December 1998 

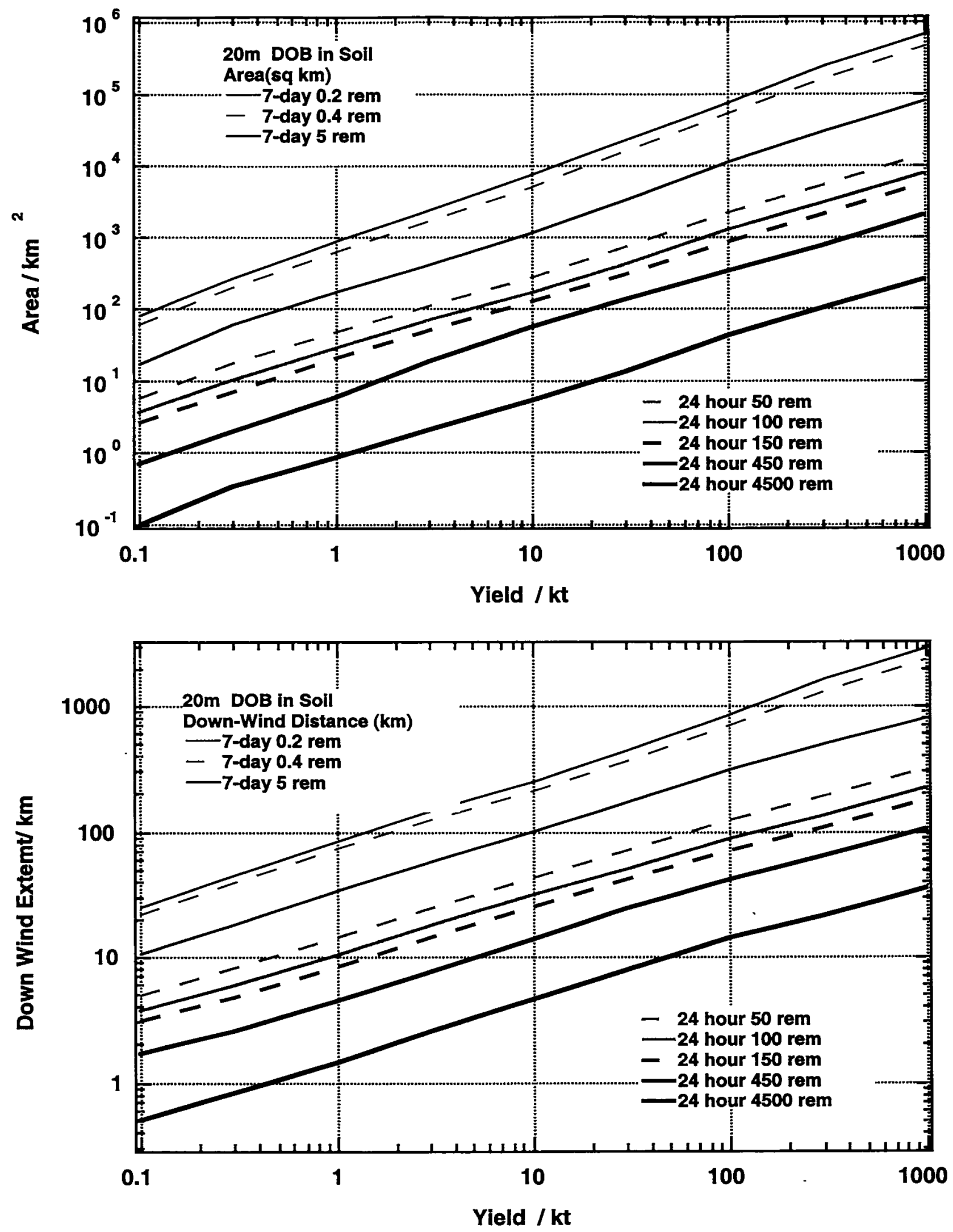

Figure 1 and Figure 2: Area and down wind extent for the indicated doses for nuclear detonations buried in 20 meters of soil 

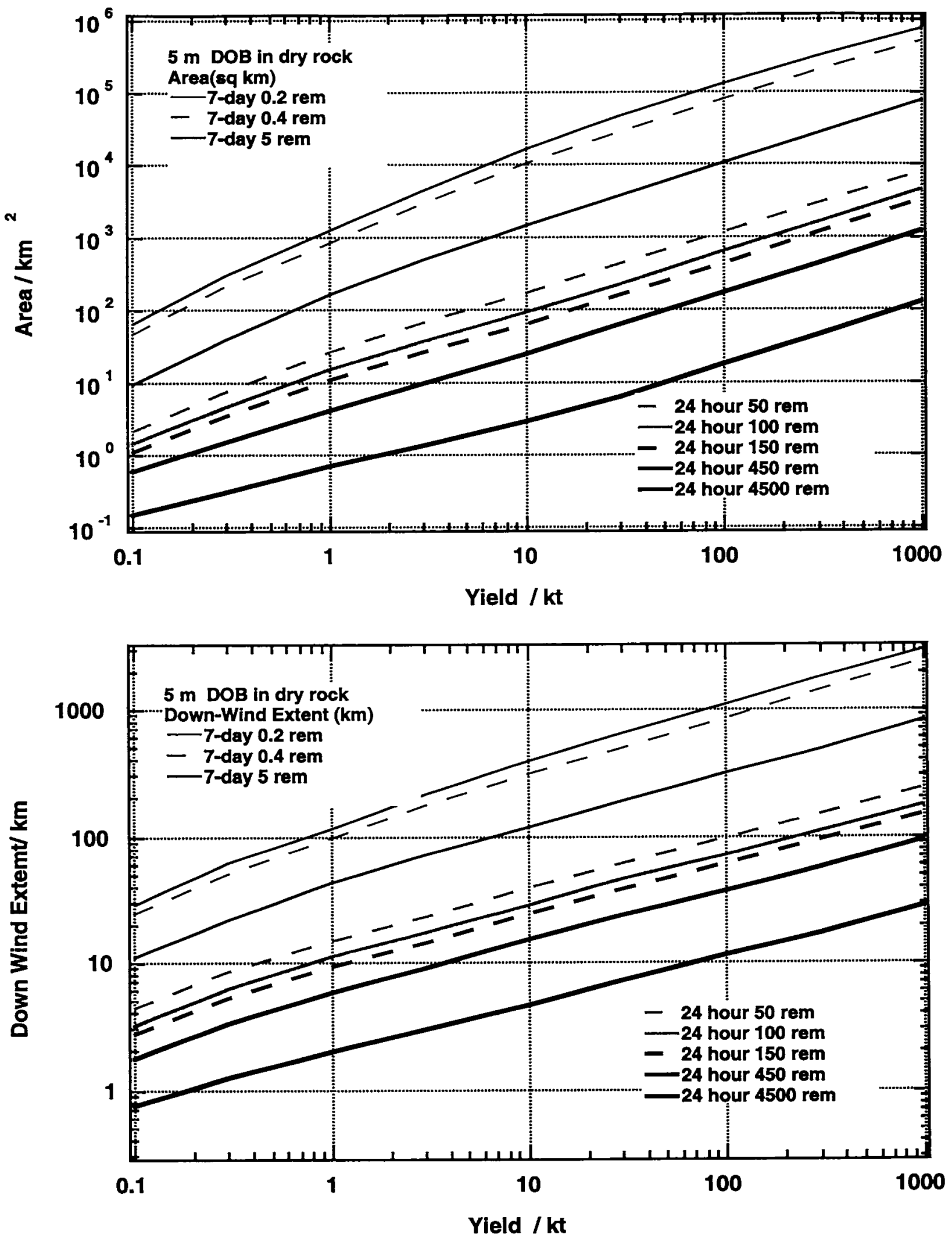

Figure 3 and Figure 4: Area and down wind extent for the indicated doses for nuclear detonations buried in 5 meters of hard, dry rock. 


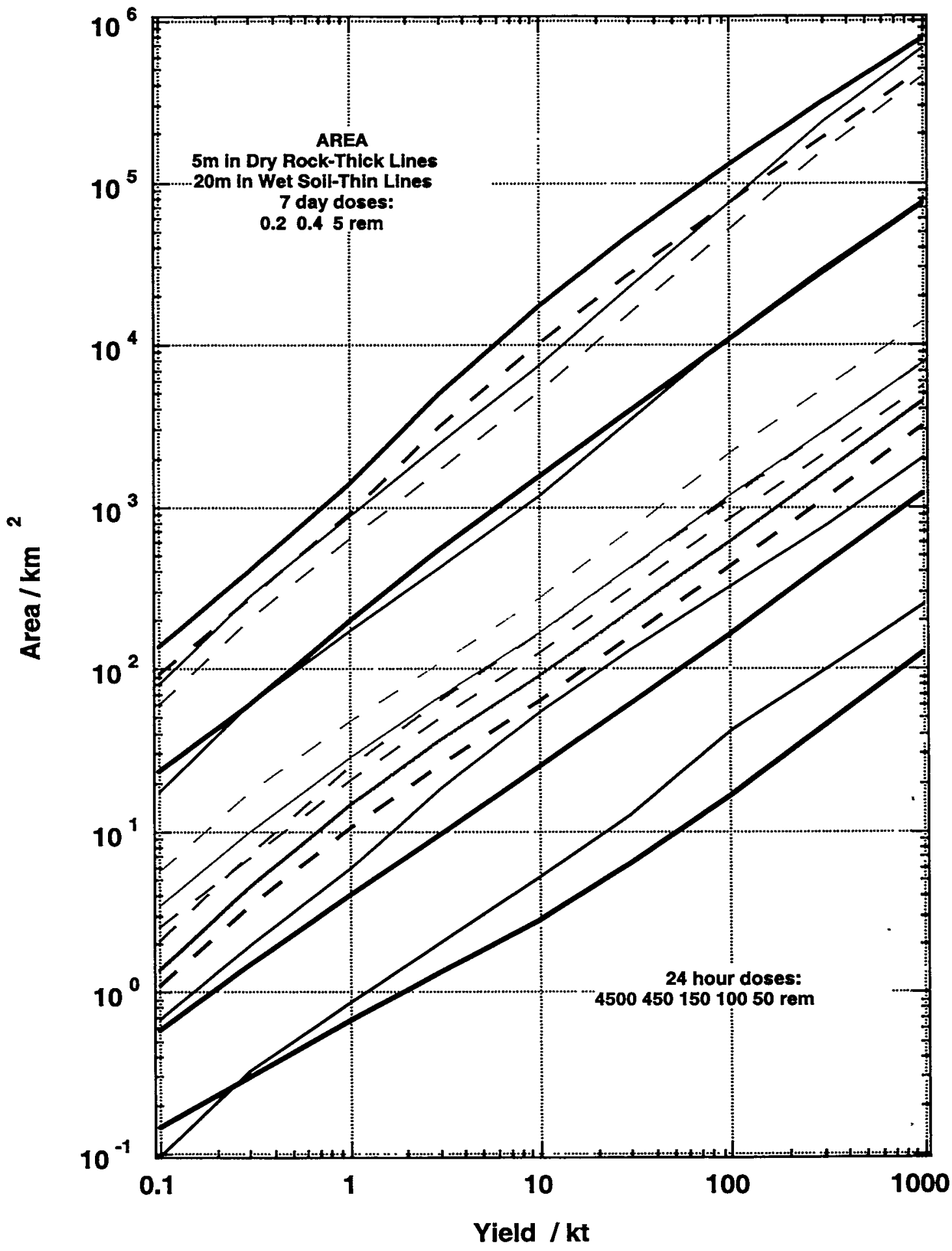

Figure 5. A combined plot that shows the areas for both the rock (bold line) and soil (thin line) scenarios considered. The colors represent the same doses as in figures 1 through 4. 


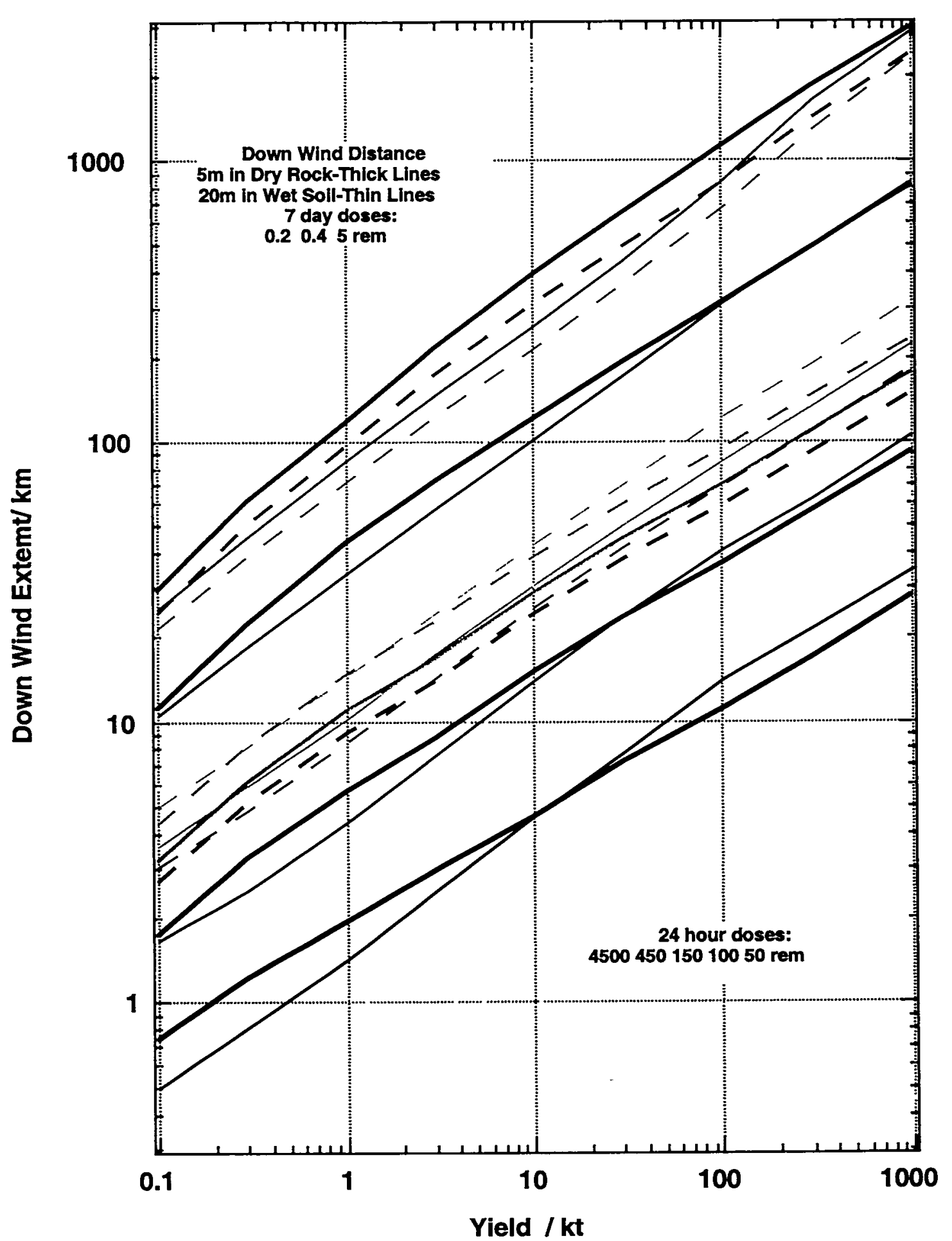

Figure 6. A combined plot that shows the down-wind extents for both the rock (bold line) and soil (thin line) scenarios considered. The colors represent the same doses as in figures 1 through 4. 


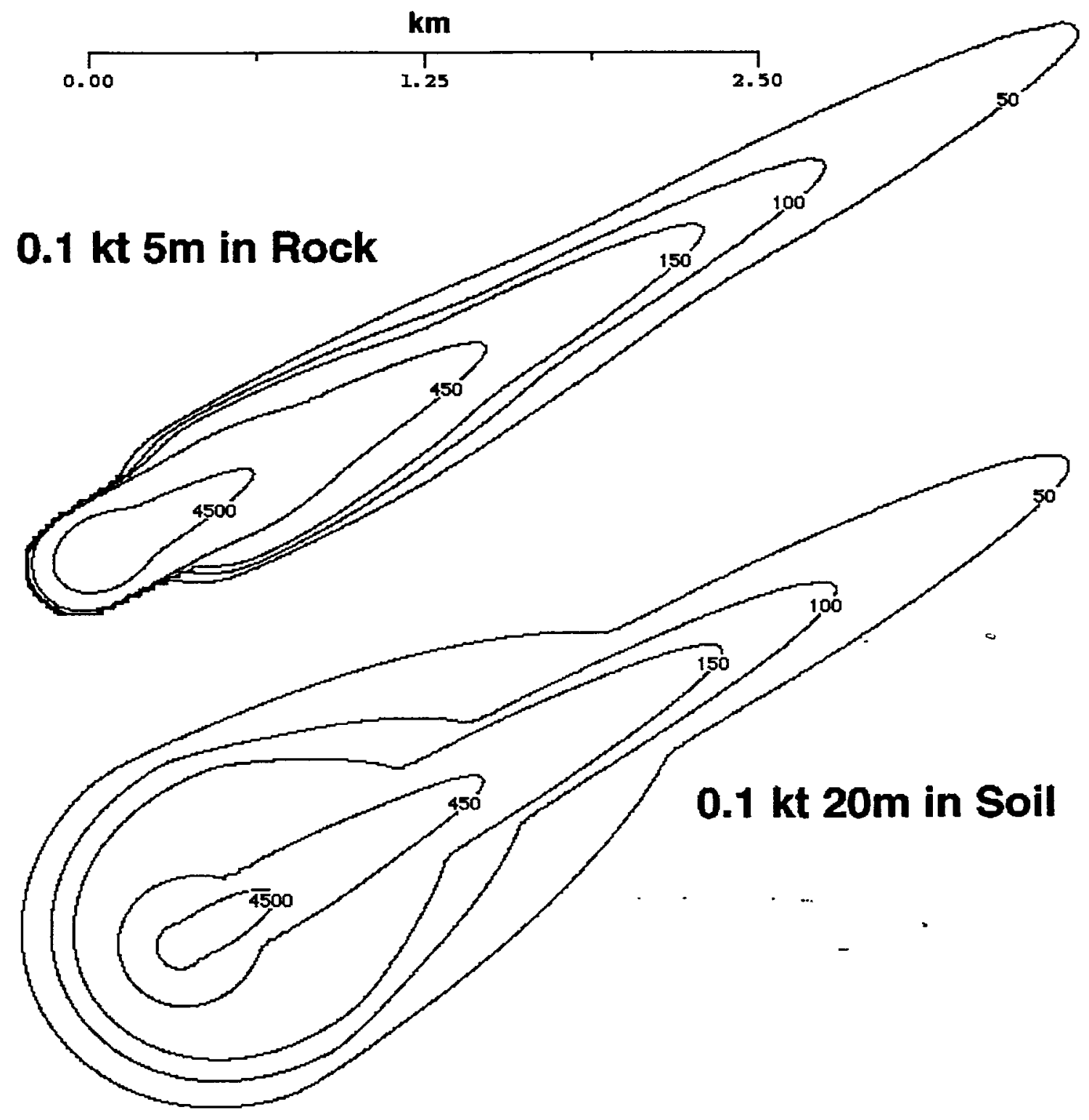

Figure 7: High dose 24-hour fallout contours for a 0.1 kt nuclear detonation emplaced in 5 meters of rock and under 20 meters of soil. 


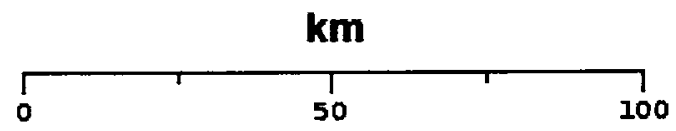

\section{Mt $5 \mathrm{~m}$ in Rock}

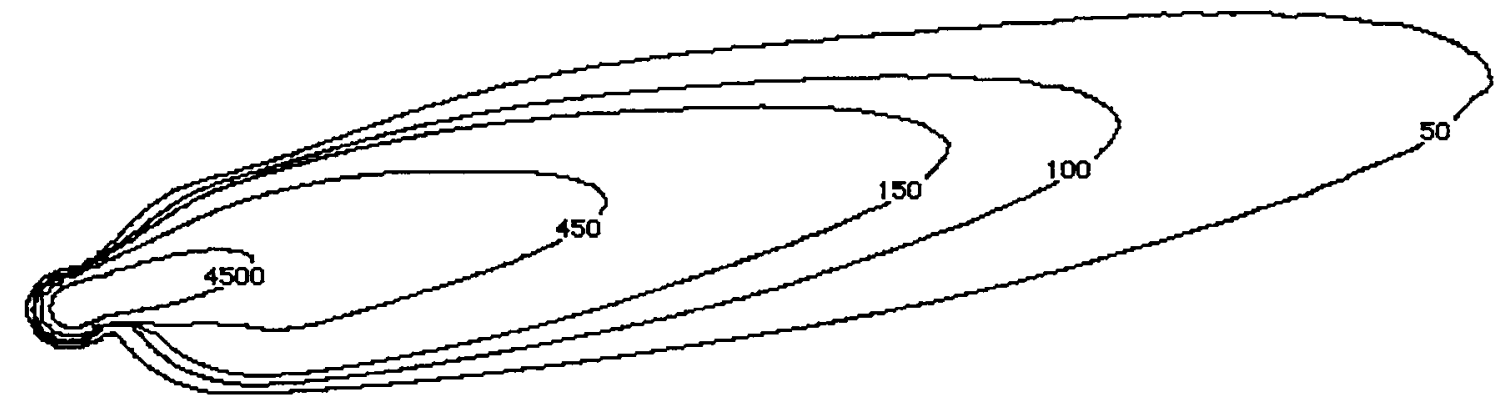

\section{$1 \mathrm{Mt} 20 \mathrm{~m}$ in Soil}

Figure 8: High dose 24-hour fallout contours for a 1 Mt nuclear detonation emplaced in 5 meters of rock and under 20 meters of soil. 